\title{
Diferenciação analítica de vinhos-base para espumantes de duas regiões vitícolas do Rio Grande do Sul
}

\author{
Analytical differentiation of the base wines for sparkling from two viticultural regions of \\ Rio Grande do Sul
}

\author{
Naira Poerner ${ }^{\mathrm{I} *}$ Eliseu Rodrigues ${ }^{\mathrm{II}}$ Paulo Gustavo Celso ${ }^{\mathrm{III}}$ Vitor Manfroi ${ }^{\mathrm{I}}$ \\ Plinho Francisco Hertz ${ }^{\mathrm{I}}$
}

\section{RESUMO}

Os objetivos deste trabalho foram caracterizar $e$ diferenciar vinhos-base para espumante (cultivares 'Chardonnay' $e$ 'Pinot Noir') provenientes da Serra do Nordeste e Serra do Sudeste do Rio Grande do Sul por meio de parâmetros físico-químicos e elementos minerais combinados com técnicas de análise multivariada. Foram analisados nove parâmetros físico-químicos (densidade, grau alcoólico, extrato seco total, extrato seco reduzido, acidez total, acidez volátil, acidez fixa, $\mathrm{pH}$ e açúcares redutores), por espectrofotometria no infravermelho, e 11 elementos minerais (Al, B, Ba, Ca, Cu, $\mathrm{Fe}, \mathrm{K}, \mathrm{Mg}, \mathrm{Mn}$, Na e Sr), por espectrometria de emissão ótica com plasma indutivamente acoplado (ICP-OES). Os elementos encontrados em maiores concentrações foram $\mathrm{K}, \mathrm{Mg}$ e Ca e, em menores concentrações, Ba, Fe, Sr e Al. A Análise de Componentes Principais (ACP) mostrou que há uma tendência natural de separação entre os vinhos-base da Serra do Nordeste e Serra do Sudeste. Por meio da Análise Discriminante (AD) foram obtidos cinco descritores ( $\mathrm{Mg}, \mathrm{Ca}, \mathrm{Mn}, \mathrm{pH}$ e grau alcoólico) para os vinhos-base da cultivar 'Chardonnay' e seis descritores (B, $\mathrm{Mn}, \mathrm{Fe}, \mathrm{Na}, \mathrm{pH}$ e acidez volátil) para a cultivar 'Pinot Noir', com capacidade de classificar as amostras de vinhos-base de acordo com a origem geográfica. Os elementos $\mathrm{Mn}$ e $\mathrm{Mg}$ parecem ser aqueles com maior capacidade de discriminação entre os vinhos-base da Serra do Nordeste e Serra do Sudeste.

Palavras-chave: vinho espumante, parâmetros físico-químicos, composição mineral, origem geográfica.

\begin{abstract}
This research describes the base wines characterization and differentiation (cultivars Chardonnay and Pinot Noir) from Serra do Nordeste and Serra do Sudeste of Rio Grande do Sul by physicochemical parameters and mineral elements followed by multivariate statistical analysis. Nine physicochemical parameters (density, alcoholic content, total dry extract, reduced dry extract, total acidity, volatile acidity, fix acidity, $\mathrm{pH}$ and reducing sugars) were analyzed by infrared spectrofotometry and eleven mineral elements ( $A l, B, B a, C a$, $\mathrm{Cu}, \mathrm{Fe}, \mathrm{K}, \mathrm{Mg}, \mathrm{Mn}, \mathrm{Na}$ and $\mathrm{Sr}$ ) by inductively coupled plasma optical emission spectrometry (ICP-OES). Elements $\mathrm{K}, \mathrm{Mg}$, and $\mathrm{Ca}$ were founded in higher concentrations and $\mathrm{Ba}, \mathrm{Fe}, \mathrm{Sr}$, and Al were founded in lower ones. Principal Component Analysis (PCA) showed a natural separation tendency between wines from Serra do Nordeste and Serra do Sudeste. Five descriptors were obtained by Discriminant Analysis (DA) for base wines from cultivar Chardonnay ( $\mathrm{Mg}, \mathrm{Ca}, \mathrm{Mn}, \mathrm{pH}$ and alcoholic content) and six descriptors for base wines from cultivar Pinot Noir (B, Mn, Fe, Na, $\mathrm{pH}$ and volatile acidity). These descriptors were capable to classify samples of base wines according to geographical origin. Mn and Mg elements seem to be the elements with higher discrimination capacity between base wines from northwest Serra and Southeast Serra.
\end{abstract}

Key words: sparkling wine, physicochemical parameters, mineral composition, geographical origin.

Instituto de Ciência e Tecnologia de Alimentos, Universidade Federal do Rio Grande do Sul (UFRGS), Porto Alegre, RS, Brasil. Rua Fernando Antônio Moreno Abdalla, Cidade Universitária, 13083-340, Campinas, SP, Brasil. E-mail: nairapoernersa@gmail.com. *Autor para correspondência.

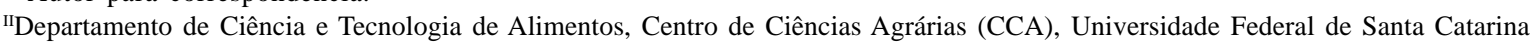
(UFSC), Florianópolis, SC, Brasil.

IIILaboratório de Análise de Bebidas do Laboratório Nacional Agropecuário (LANAGRO), Ministério da Agricultura, Pecuária e Abastecimento (MAPA), Porto Alegre, RS, Brasil. 


\section{INTRODUÇÃO}

O Estado do Rio Grande do Sul é o principal pólo de produção de uvas do Brasil, possuindo uma área de 49.816 hectares, o que representa 60,3\% da área total de cultivo de uvas do Brasil. Esse Estado responde por mais de $90 \%$ da produção brasileira de vinhos e sucos de uva (MELLO, 2008). Nos últimos anos, houve uma ampliação da sua área de cultivo, principalmente com vinhedos Vitis vinifera L., para elaboração de vinhos finos, com destaque para os vinhos espumantes. Além da tradicional região produtora Serra do Nordeste (conhecida também como Serra Gaúcha), há a implantação de cultivares de uvas viníferas na Serra do Sudeste, a qual abrange os Municípios de Encruzilhada do Sul e Pinheiro Machado. Essas duas regiões apresentam características de produção distintas, sendo a primeira marcada pela agricultura familiar e a segunda pela mecanização (PROTAS et al., 2002). Quanto às condições climáticas, ambas apresentam semelhante classe de clima (temperado quente, úmido e de noites temperadas). No entanto, apresentam diferenças em relação ao material geológico que dá origem aos solos, uma vez que o material de origem dos solos da Serra do Nordeste é predominantemente o basalto, e o da Serra do Sudeste são as rochas de origem graníticas (STRECK et al., 2008). De acordo com TONIETTO \& CARBONNEAU (2004), essas diferenças dos fatores naturais e humanos são suficientes para gerar vinhos com qualidade e tipicidade próprias.

Os vinhos finos nacionais têm apresentado grande evolução qualitativa, reconhecida nacional e até internacionalmente, o que pode ser comprovado pelo bom desempenho de algumas vinícolas brasileiras em concursos internacionais de vinhos. Na categoria dos vinhos finos, destaca-se o vinho espumante gaúcho, um produto de qualidade reconhecida e que apresenta grandes perspectivas de mercado (PROTAS et al., 2002). É elaborado a partir de uma segunda fermentação alcoólica conduzida em um vinho-base, apresentando características químicas e sensoriais relacionadas ao vinho-base utilizado. A sua composição é influenciada por diversos fatores correspondentes à área específica de produção, como a cultivar de uva, o solo e clima, a cultura, a levedura, o processo de elaboração do vinho, o transporte e o armazenamento (JOS, et al., 2004; SPERKOVÁ \& SUCHÁNEK, 2005).

O progresso das técnicas de análise multielementar tem aumentado rapidamente nos últimos anos e, de modo paralelo, também o número de trabalhos que visam a caracterizar e diferenciar os vinhos de acordo com o local de produção. Esses trabalhos utilizam diferentes parâmetros químicos, entre eles, a análise de elementos minerais. Apesar de vários estudos sobre a diferenciação de vinhos terem sido publicados, são escassos aqueles que avaliaram os vinhos-base empregados na elaboração de vinhos espumantes. Assim, a proposta deste trabalho foi caracterizar e diferenciar os vinhos-base das cultivares 'Chardonnay' e 'Pinot Noir' provenientes da Serra do Nordeste e da Serra do Sudeste do Rio Grande do Sul. Para tanto, parâmetros físico-químicos e composição mineral foram determinados conjuntamente com a aplicação de técnicas de análise multivariada.

\section{MATERIAL E MÉTODOS}

Foram analisadas 66 amostras de vinhosbase das cultivares 'Chardonnay' e 'Pinot Noir' da safra de 2008 provenientes de duas principais regiões de produção de uvas Vitis vinifera L. do Estado do Rio Grande do Sul: a Serra do Nordeste (SN) e a Serra do Sudeste (SS). Do total de amostras coletadas, 31 foram da cultivar 'Chardonnay' (21 da SN e 10 da SS) e 35 da cultivar 'Pinot Noir' (17 da SN e 18 da SS).

Para as análises, as amostras foram desgaseificadas previamente em equipamento ultrassom (USC-1400 Unique). Os parâmetros grau alcoólico (GA) e extrato seco total (EST) foram determinados por espectrofotometria no infravermelho próximo (NIR) por meio do aparelho Alcolyser Wine (Anton Paar, Graz, Áustria) acoplado a um densímetro digital, o DMA 4500 (Anton Paar, Graz, Áustria). Acidez total (AT) e volátil (AV), pH e açúcares redutores (AR) foram determinados por espectrofotometria no infravermelho com transformada de Fourier (FTIR) utilizando o aparelho WineScan (Foss, Dinamarca). A acidez fixa (AF) e o extrato seco reduzido (ESR) foram determinados a partir de cálculo (BRASIL, 2005).

A determinação de $\mathrm{Al}, \mathrm{B}, \mathrm{Ba}, \mathrm{Ca}, \mathrm{Cu}, \mathrm{Fe}, \mathrm{K}$, $\mathrm{Mg}, \mathrm{Mn}, \mathrm{Na}$ e Sr foi realizada empregando um espectrômetro de emissão ótica com plasma indutivamente acoplado simultâneo (ICP-OES), modelo MPX-CCD da Varian (Mulgrave, Austrália), com tocha na configuração radial.

Para a quantificação, as amostras de vinhosbase foram desgaseificadas previamente em equipamento ultrassom (USC-1400 Unique), sendo realizada a análise direta dos elementos $\mathrm{Al}, \mathrm{B}, \mathrm{Ba}, \mathrm{Cu}$, Fe, Mn e Sr. Para determinação de Ca, K, Mg e Na, as amostras foram diluídas em um fator de 10 vezes em ácido nítrico $1 \mathrm{~mol} \mathrm{~L}^{-1}$. A quantificação foi realizada pelo método de calibração externa utilizando solução padrão multielementar em etanol $12 \% \mathrm{v} \mathrm{v}^{-1}$ (faixa de concentração de 0,1 a $10 \mathrm{mg} \mathrm{L}^{-1}$ ) e etanol $1,2 \% \mathrm{v} \mathrm{v}^{-1}$ 
(faixa de concentração de 1 a $100 \mathrm{mg} \mathrm{L}^{-1}$ ), para análise das amostras sem diluição e das amostras diluídas, respectivamente.

As determinações foram realizadas em triplicata, e os dados estão apresentados como média \pm desvio-padrão. A avaliação das diferenças entre as médias foi realizada pela aplicação da ANOVA, considerando o nível de 5\% de significância. As técnicas multivariadas Análise de Componentes Principais (ACP) e Análise Discriminante (AD) foram executadas em dados previamente autoescalonados. Todas as análises estatísticas foram realizadas empregando o software Statistica $6.0^{\circledR}$.

\section{RESULTADOS E DISCUSSÃO}

A tabela 1 apresenta os resultados das análises físico-químicas e de composição mineral das amostras de vinhos-base das cultivares 'Chardonnay' e 'Pinot Noir' provenientes da SN e SS. A análise de variância (ANOVA) indica que os parâmetros físicoquímicos D, EST, ESR, AV e AR não apresentaram diferenças significativas $(\mathrm{P}>0,05)$ entre os vinhos-base da cultivar 'Chardonnay' das duas regiões. De modo contrário, foram encontradas diferenças significativas $(\mathrm{P}<0,05)$ para os parâmetros $\mathrm{GA}, \mathrm{AT}, \mathrm{AF}$ e $\mathrm{pH}$, apresentando valores maiores as amostras da SS, com exceção do pH. As amostras de vinhos-base da cultivar 'Pinot Noir' da SN e SS não apresentaram diferenças significativas $(\mathrm{P}>0,05)$ para D, EST, ESR, AT eAF. Porém, apresentaram diferenças significativas $(\mathrm{P}<0,05)$ para $\mathrm{o}$ $\mathrm{GA}, \mathrm{AV}$, pH e AR, sendo os teores maiores nos vinhosbase da SS. Em geral, os resultados das análises físicoquímicas são semelhantes aos obtidos por STEFENON (2006), que avaliou cinco amostras de vinhos-base dessas mesmas regiões.

Conforme pode ser observado na tabela 1 , não há diferença significativa $(\mathrm{P}>0,05)$ entre as amostras de vinhos-base da cultivar 'Chardonnay' para os elementos $\mathrm{B}, \mathrm{Ba}, \mathrm{Cu}, \mathrm{K}$ e $\mathrm{Na}$, bem como para os elementos $\mathrm{Al}$, Cu, Sr e K, nas amostras de vinhos-base da cultivar 'Pinot Noir' da SN e SS. O K foi o elemento encontrado em maiores concentrações em todas as amostras de vinhos-base. Para ambas cultivares, não foi observada diferença significativa $(P>0,05)$ no teor desse elemento entre as regiões estudadas. No caso do conteúdo de $\mathrm{Mn}, \mathrm{Mg}, \mathrm{Fe}$ e Ca, há diferença significativa $(\mathrm{P}<0,05)$ entre os vinhos-base para ambas

Tabela 1 - Parâmetros físico-químicos e elementos minerais ( $\left.\mathrm{mg} \mathrm{L}^{-1}\right)$ de vinhos-base da Serra do Nordeste (SN) e da Serra do Sudeste (SS).

\begin{tabular}{|c|c|c|c|c|}
\hline \multirow{2}{*}{ Parâmetro avaliado } & \multicolumn{2}{|c|}{-------Vinho-base da cultivar ‘Chardonnay’--------- } & \multicolumn{2}{|c|}{-------Vinho-base da cultivar 'Pinot Noir'-------- } \\
\hline & $\mathrm{SN}(\mathrm{n}=21)$ & SS $(n=10)$ & $\mathrm{SN}(\mathrm{n}=17)$ & SS $(n=18)$ \\
\hline $\mathrm{D}$ & $0,9909 \pm 0,0009^{\mathrm{A}}$ & $0,9901 \pm 0,0015^{\mathrm{A}}$ & $0,9915 \pm 0,0007^{\mathrm{a}}$ & $0,9912 \pm 0,0008^{\mathrm{a}}$ \\
\hline $\mathrm{GA}\left(\% \mathrm{v} \mathrm{v}^{-1}\right)$ & $11,40 \pm 0,46^{\mathrm{A}}$ & $12,29 \pm 0,82^{\mathrm{B}}$ & $11,00 \pm 0,20^{\mathrm{a}}$ & $11,55 \pm 0,61^{b}$ \\
\hline $\operatorname{EST}\left(\mathrm{g} \mathrm{L}^{-1}\right)$ & $20,28 \pm 1,72^{\mathrm{A}}$ & $20,98 \pm 1,62^{A}$ & $20,65 \pm 1,33^{\text {a }}$ & $21,43 \pm 1,03^{\mathrm{a}}$ \\
\hline $\operatorname{ESR}\left(\mathrm{g} \mathrm{L}^{-1}\right)$ & $19,70 \pm 1,37^{\mathrm{A}}$ & $20,58 \pm 1,42^{\mathrm{A}}$ & $19,93 \pm 1,14^{\mathrm{a}}$ & $20,20 \pm 1,19^{\text {a }}$ \\
\hline $\mathrm{AT}\left(\mathrm{meq} \mathrm{L} \mathrm{L}^{-1}\right)$ & $87,95 \pm 15,30^{\mathrm{A}}$ & $99,89 \pm 11,96^{\text {В }}$ & $92,69 \pm 14,97^{\text {a }}$ & $94,64 \pm 14,83^{a}$ \\
\hline $\mathrm{AV}\left(\mathrm{meq} \mathrm{L} \mathrm{L}^{-1}\right)$ & $10,49 \pm 4,18^{\mathrm{A}}$ & $10,75 \pm 4,07^{\mathrm{A}}$ & $12,31 \pm 2,54^{\text {a }}$ & $13,79 \pm 0,31^{b}$ \\
\hline $\mathrm{AF}\left(\mathrm{meq} \mathrm{L}^{-1}\right)$ & $77,46 \pm 12,80^{\mathrm{A}}$ & $89,15 \pm 10,18^{\text {В }}$ & $80,38 \pm 12,59^{a}$ & $80,85 \pm 14,76^{a}$ \\
\hline $\mathrm{pH}$ & $3,46 \pm 0,09^{\mathrm{A}}$ & $3,41 \pm 0,16^{\mathrm{B}}$ & $3,35 \pm 0,11^{\mathrm{a}}$ & $3,44 \pm 0,13^{b}$ \\
\hline $\mathrm{AR}\left(\mathrm{g} \mathrm{L}^{-1}\right)$ & $1,58 \pm 0,42^{\mathrm{A}}$ & $1,40 \pm 0,22^{\mathrm{A}}$ & $1,71 \pm 0,27^{\mathrm{a}}$ & $2,23 \pm 0,47^{b}$ \\
\hline Alumínio & $0,32 \pm 0,15^{\mathrm{A}}$ & $0,16 \pm 0,11^{\text {в }}$ & $0,33 \pm 0,26^{a}$ & $0,30 \pm 0,33^{a}$ \\
\hline Boro & $3,01 \pm 0,16^{\mathrm{A}}$ & $3,03 \pm 0,60^{\mathrm{A}}$ & $2,82 \pm 0,67^{\mathrm{a}}$ & $3,07 \pm 0,30^{b}$ \\
\hline Bário & $0,24 \pm 0,05^{\mathrm{A}}$ & $0,26 \pm 0,18^{A}$ & $0,21 \pm 0,04^{\mathrm{a}}$ & $0,16 \pm 0,04^{b}$ \\
\hline Cobre & $0,57 \pm 0,42^{\mathrm{A}}$ & $0,57 \pm 0,42^{\mathrm{A}}$ & $0,76 \pm 0,43^{a}$ & $0,70 \pm 0,39^{\mathrm{a}}$ \\
\hline Ferro & $0,44 \pm 0,48^{\mathrm{A}}$ & $0,23 \pm 0,17^{\text {в }}$ & $0,23 \pm 0,12^{\mathrm{a}}$ & $0,15 \pm 0,04^{b}$ \\
\hline Manganês & $2,19 \pm 0,44^{\mathrm{A}}$ & $3,52 \pm 1,79^{\mathrm{B}}$ & $2,00 \pm 0,25^{a}$ & $3,23 \pm 0,61^{b}$ \\
\hline Estrôncio & $0,38 \pm 0,09^{\mathrm{A}}$ & $0,32 \pm 0,04^{\mathrm{B}}$ & $0,29 \pm 0,08^{a}$ & $0,30 \pm 0,06^{\mathrm{a}}$ \\
\hline Cálcio & $72,55 \pm 11,82^{\mathrm{A}}$ & $60,16 \pm 7,29^{\text {в }}$ & $64,95 \pm 10,41^{\mathrm{a}}$ & $57,83 \pm 6,11^{\mathrm{b}}$ \\
\hline Potássio & $676,82 \pm 177,42^{\text {A }}$ & $664,11 \pm 246.82^{\mathrm{A}}$ & $583,68 \pm 110,48^{a}$ & $591,53 \pm 33,31^{a}$ \\
\hline Magnésio & $61,95 \pm 4,28^{\mathrm{A}}$ & $75,69 \pm 4,50^{\text {B }}$ & $52,24 \pm 5,41^{a}$ & $60,52 \pm 6,76^{b}$ \\
\hline Sódio & $9,23 \pm 13,21^{A}$ & $9,78 \pm 5,76^{\mathrm{A}}$ & $5,55 \pm 1,03^{\mathrm{a}}$ & $8,50 \pm 1,90^{b}$ \\
\hline
\end{tabular}

Os dados estão apresentados como média \pm desvio-padrão.

D: densidade; GA: grau alcoólico; EST: extrato seco total; ESR: extrato seco reduzido; AT: acidez total; AV: acidez volátil; AF: acidez fixa; AR: açúcares redutores.

* Valores seguidos de letras diferentes, na mesma linha, dentro de cada cultivar, apresentam diferença significativa ao nível de 5\% de significância. 
as cultivares da SN e SS. Os elementos Mn e Mg estão presentes em concentrações superiores nos vinhosbase da SS quando comparados aos da SN, independente da cultivar avaliada. Observa-se um comportamento inverso quando são considerados os elementos $\mathrm{Fe}$ e $\mathrm{Ca}$, ou seja, estão presentes em concentrações superiores nas amostras da SN.

A análise da matriz de correlação de Pearson (dados não apresentados) dos elementos minerais nos vinhos-base da cultivar 'Chardonnay' mostra grande número de valores com coeficiente de correlação $\left(r^{2}\right)>0,70$, destacando-se a alta correlação negativa entre os elementos Cu e Fe (-0,92); Mn e B (-0,91); Mn e Ba (0,89); e Mg e Ba (-0,80). Há uma forte correlação positiva entre os elementos Mn e Mg $(0,85)$; $\mathrm{Mg}$ e $\mathrm{Na}(0,84)$; B e Ba (0,82); Fe e Sr $(0,71)$; K e Ca $(0,70)$; e Mn e Na $(0,69)$. Para a cultivar 'Pinot Noir' destaca-se a correlação negativa entre Ca e Mn (-0,92); Ca e Na ($0,86)$; Cu e Mg (-0,84); Fe e Mn (-0,84); $M g$ eAl (-0,79); Ba e Mn (-0,78); e Ba e Na (-0,71). Com correlação positiva para essa cultivar, destaca-se os elementos $\mathrm{Al}$ e Cu (0,98); B e Sr (0,94); Fe e Ca (0,94); Mn e Na (0,98); Mg e Na $(0,85)$; e Mn e Mg $(0,77)$. Em ambas as cultivares, os elementos Mg, Na e Mn apresentaram uma correlação positiva, o que pode estar relacionado com a origem litofílica desses elementos (KMENT et al., 2005).

Apesar de informações interessantes serem obtidas pela aplicação de técnicas univariadas como a ANOVA, essa técnica não permitiu a diferenciação entre os vinhos-base das duas regiões. Assim, as técnicas de análise multivariada ACP AD foram realizadas para diferenciação dos vinhos-base de acordo com o local de produção. As variáveis consideradas para essas análises foram somente aquelas que apresentaram diferenças significativas $(\mathrm{P}<0,05)$ na ANOVA.

A ACP foi aplicada visando reduzir a dimensão dos dados em componentes principais e visualizar suas similaridades. Essa técnica gera um novo grupo de variáveis, obtido através da melhor combinação linear dos parâmetros originais, que são os principais contribuintes para a variância.

Nas amostras de vinhos-base da cultivar 'Chardonnay', foram avaliados 10 parâmetros (GA, AT, AF, pH, Al, Fe, Mn, Sr, Ca e Mg). Para os vinhos-base da cultivar 'Pinot Noir', foram considerados 11 parâmetros (GA, AV, pH, AR, B, Ba, Fe, Mn, Ca, Mg e $\mathrm{Na}$ ). Os demais parâmetros não foram considerados na matriz dos dados, pois não apresentaram contribuições significativas para diferenciar os vinhos-base de acordo com a origem geográfica. A tabela 2 apresenta a ACP executada com o objetivo de diferenciar os vinhosbase da cultivar 'Chardonnay' provenientes da SN e SS. Como pode ser observado, os quatro primeiros CPs respondem por 93,23\% do total da variância. O CP1 responde por 37,23\% da variância total, sendo explicado principalmente por Al, Mg, Mn, AT e AF.

A figura 1A apresenta o gráfico da projeção dos vinhos-base da cultivar 'Chardonnay' pelos CP1 e CP2. Nesse gráfico, verifica-se uma separação natural entre os vinhos-base da cultivar 'Chardonnay' provenientes das duas regiões estudadas que pode ser explicada pelos valores de loadings da tabela 2. Os parâmetros Mg (-0.683618), Mn (-0.621966), AT (0.833815) e AF (-0.827400) apresentam loadings negativos, separando os vinhos-base das duas regiões

Tabela 2 - Contribuição dos parâmetros para os loadings dos componentes principais, eingenvalues e explicação da variância - cultivar 'Chardonnay'.

\begin{tabular}{|c|c|c|c|c|}
\hline \multirow{2}{*}{ Elemento } & \multirow[b]{2}{*}{ CP1 } & \multirow[b]{2}{*}{$\mathrm{CP} 2$} & \multirow[b]{2}{*}{ CP3 } & \multirow[b]{2}{*}{$\mathrm{CP} 4$} \\
\hline & & & & \\
\hline $\mathrm{Al}$ & 0,694798 & 0,422273 & $-0,418462$ & $-0,332808$ \\
\hline $\mathrm{Fe}$ & 0,512290 & 0,480865 & 0,484503 & $-0,485447$ \\
\hline $\mathrm{Mn}$ & $-0,621966$ & 0,217898 & $-0,312634$ & $-0,611385$ \\
\hline $\mathrm{Sr}$ & 0,380742 & 0,774011 & 0,397801 & $-0,077291$ \\
\hline $\mathrm{Ca}$ & 0,334061 & 0,503828 & 0,578696 & 0,383469 \\
\hline $\mathrm{Mg}$ & $-0,683618$ & $-0,252483$ & 0,565520 & $-0,347379$ \\
\hline GA & $-0,321623$ & $-0,653101$ & 0,641131 & $-0,120053$ \\
\hline AT & $-0,833815$ & 0,451530 & $-0,171285$ & 0,134213 \\
\hline $\mathrm{AF}$ & $-0,827400$ & 0,526861 & 0,004656 & 0,093153 \\
\hline $\mathrm{pH}$ & 0,619940 & $-0,705640$ & $-0,069123$ & $-0,138907$ \\
\hline Eigenvalues & 3,723526 & 2,779640 & 1,765728 & 1,054305 \\
\hline \% variância & 37.23526 & 27,79640 & 17,65728 & 10,54305 \\
\hline \% variância acumulada & 37.2353 & 65,0317 & 82,6889 & 93,2320 \\
\hline
\end{tabular}




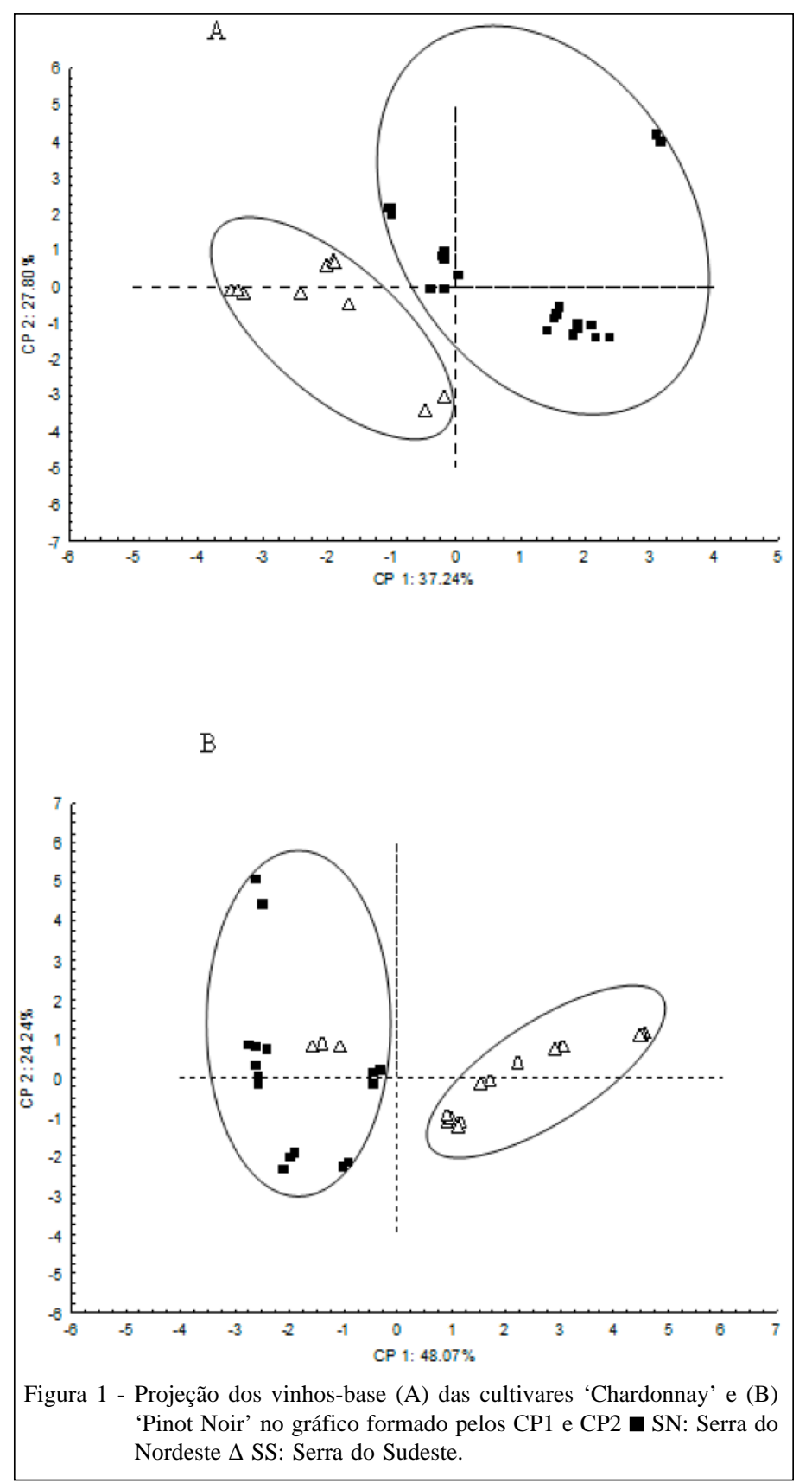

Conforme pode ser observado na tabela 3, os três primeiros CPs explicam a maior parte da variância $(83,44 \%)$ dos vinhos-base da cultivar 'Pinot Noir'. O CP1, que responde por 48,07\% da variância, é explicado principalmente por $\mathrm{Mn}, \mathrm{Na}, \mathrm{Mg}$, GA e AR. Na figura 1B, pode-se observar que há uma tendência de separação entre os vinhos-base da cultivar 'Pinot Noir' provenientes da SN e SS, pois os vinhosbase da SS quase em sua totalidade apresentam escores positivos para CP1, em razão das maiores concentrações de $\mathrm{Mn}$, $\mathrm{Na}, \mathrm{Mg}, \mathrm{GA}$ e AR (loadings positivos). A separação não foi completa pelo fato de que as três amostras da SS apresentaram um padrão de composição diferente da região de origem e similar aos da $\mathrm{SN}$.

Os elementos $\mathrm{Mn}$ e $\mathrm{Mg}$ apresentaram influência significativa no CP1 das duas cultivares, sendo essa participação explicada pelo fato de as amostras provenientes da SS apresentarem concentrações superiores desses elementos. Segundo RIZZON et al. (2008), os vinhos da SN apresentam concentrações inferiores de Mg que outras regiões vitícolas em decorrência da menor presença desse elemento no solo. Estudo desenvolvido por KMENT et al. (2005) confirma que há uma correlação positiva $(0,616)$ entre a concentração do $\mathrm{Mg}$ presente no solo e no vinho, destacando que o conteúdo desse elemento é influenciado fortemente pela composição da matriz rochosa. FRÍAS et al. (2001) descreveram que o Mg é um dos elementos mais empregados na discriminação de origem de vinhos. Por sua vez, a concentração superior de Mn pode ser explicada pela maior acidez do solo da SS, visto que os vinhedos cultivados nesse

em razão das maiores concentrações de Mg e Mn dos maiores valores de AT e AF nos vinhos-base da SS. Os vinhos-base da SN são diferenciados por apresentarem maiores concentrações de $\mathrm{Al}$ (loading positivo). De acordo com MARENGO \& ACETO (2003), o Al pertence ao grupo de elementos cuja concentração no vinho não é influenciada pelo ciclo de produção, sendo afetada pela contribuição mineral do solo ou pela capacidade de absorção por meio do sistema radicular da videira. tipo de solo absorvem maior quantidade de Mn pela raiz da videira (RIZZON et al., 2008; STRECK et al., 2008).

AAD foi aplicada visando obter descritores químicos que pudessem classificar corretamente os vinhos-base das duas cultivares estudadas de acordo com a região de produção. A AD aplicada aos dados dos vinhos-base da cultivar 'Chardonnay' mostrou que os elementos Mg, Ca e Mn e os parâmetros GA e pH permitiram a diferenciação completa das amostras dessas duas regiões. Através desses descritores, foram 
Tabela 3 - Contribuição dos parâmetros para os loadings dos componentes principais, eingenvalues e explicação da variância - cultivar 'Pinot Noir'.

\begin{tabular}{llll}
\hline & & & \\
Elemento & \multicolumn{1}{c}{ CP1 } & \multicolumn{1}{c}{ CP2 } & \multicolumn{1}{c}{ CP3 } \\
\hline B & 0,176758 & 0,675411 & 0,378612 \\
Ba & $-0,654139$ & $-0,035446$ & 0,600110 \\
Fe & $-0,633820$ & 0,693196 & $-0,194603$ \\
Mn & 0,947743 & $-0,056055$ & $-0,120363$ \\
Ca & $-0,572509$ & 0,752758 & 0,198605 \\
Mg & 0,833954 & 0,449039 & $-0,017827$ \\
Na & 0,926816 & 0,040618 & $-0,242991$ \\
GA & 0,850604 & 0,247293 & $-0,036464$ \\
AR & 0,744368 & 0,048394 & 0,554969 \\
AV & 0,358970 & $-0,625243$ & 0,587417 \\
pH & 0,489971 & 0,707849 & 0,157542 \\
Eigenvalue & 5,287860 & 2,666505 & 1,333842 \\
\% variância & 48,07145 & 24,24096 & 12,12584 \\
\% variância acumulada & 48,07145 & 72,3124 & 84,4382 \\
\hline & & & \\
\hline
\end{tabular}

obtidos $100 \%$ de correta classificação. Para os vinhosbase da cultivar 'Pinot Noir', foram selecionados seis descritores (B, Mn, Fe, Na, AV e pH), que também permitiram $100 \%$ de correta classificação. Esses resultados indicam que um número reduzido de variáveis contém a mesma informação que as 20 variáveis analisadas neste trabalho e podem ser usadas para identificar a origem dos vinhos-base da SN e SS. As informações obtidas na AD estão de acordo com as apresentadas pela ACP, principalmente na demonstração da importância do elemento Mn na diferenciação da origem geográfica dos vinhos-base de ambas as cultivares. A partir do potencial estimado dos elementos Mn e Mg na diferenciação dos vinhosbase das duas regiões, pode-se propor um modelo de classificação baseado nesses dois elementos. Ao ser realizada uma nova $\mathrm{AD}$ utilizando somente esses elementos, obteve-se correta classificação de 83,3\% para os vinhos-base da SS e de $100 \%$ da SN, em ambas as cultivares.

\section{CONCLUSÃO}

A ACP mostrou que há uma tendência natural de separação entre os vinhos-base provenientes da SN e SS. AAD permitiu encontrar um número reduzido de descritores químicos com a informação necessária para classificar os vinhos-base como pertencentes a uma das regiões avaliadas. Os vinhos-base da cultivar 'Chardonnay' foram classificados em um modelo a partir de cinco descritores químicos (Mg, Ca, Mn, GA e pH) e os da cultivar 'Pinot Noir' a partir de seis descritores químicos (B, Mn, Fe, $\mathrm{Na}, \mathrm{AV}$ e pH), que possibilitaram discriminação completa das amostras quanto a seu local de produção. Os elementos Mn e Mg apresentaram-se com maior potencial de discriminação entre os vinhos-base da Serra do Nordeste e Serra do Sudeste.

\section{AGRADECIMENTOS}

À Coordenação de Aperfeiçoamento de Pessoal de Nível Superior (CAPES), pelo auxílio financeiro, e às empresas Möet Hennessy do Brasil, Cooperativa Vinícola Aurora, Cooperativa Vinícola Garibaldi, Cooperativa Vitivinícola Aliança e Casa Valduga, pelo fornecimento das amostras.

\section{REFERÊNCIAS}

BRASIL. Instrução Normativa $\mathrm{n}^{\circ} 24$, de 8 de setembro de 2005. Diário Oficial da União. Aprova o Manual Operacional de Bebidas e Vinagres. Brasília: Ministério da Agricultura, Pecuária e Abastecimento, 2005.

FRÍAS S. et al. Classification and differentiation of bottled sweet wines of Canary Islands (Spain) by their metallic content. European Food Research and Technology, v.213, p.145149, 2001. Disponível em: <http:// www.springerlink.com/ content/xbdqgbeutvmk889n/fulltext.pdf $>$. Acesso em: 13 maio 2009. doi: $10.1007 / \mathrm{s} 002170100344$

JOS, A. et al. Differentiation of sparkling wines (cava and champagne) according to their mineral content. Talanta, v.63, p.377-382, 2004. Disponível em: <http://linkinghub.elsevier.com/ retrieve/pii/S0039914003006611>. Acesso em: 13 maio 2009. doi:10.1016/j.talanta.2003.11.015

KMENT, P. et al. Differentiation of Czech wines using multielement composition - A comparison with vineyard soil. Food Chemistry, v.91, p.157-165, 2005. Disponível em: $<$ http://linkinghub.elsevier.com/retrieve/pii/ S0308814604004595>. Acesso em: 13 maio 2009. doi: 10.1016/j.foodchem.2004.06.010.

MARENGO, E.; ACETO, M. Statistical investigation of the differences in the distribution of metals in Nebbiolo-based wines. Food Chemistry, v.81, p.621-630, 2003. Disponível em: $<$ http://linkinghub.elsevier.com/retrieve/pii/ S0308814602005642>. Acesso em: 13 maio 2009. doi: 10.1016/S0308-8146(02)00564-2.

MELLO, L.M.R. Vitivinicultura Brasileira: panorama 2008. Bento Gonçalves: Embrapa Uva e Vinho, 2008. 4p. (Artigo Técnico). Disponível em: <http://www.cnpuv.embrapa.br/ publica/artigos/vitbras2008.pdf>. Acesso em: 11 jun. de 2009.

PROTAS, J.F.S. et al. A vitivinicultura brasileira: realidade e perspectivas. Bento Gonçalves: Embrapa Uva e Vinho, 2002. (Artigo Técnico). Disponível em: <http://www.cnpuv.embrapa.br/ publica/artigos/vitivinicultura/>. Acesso em: $04 \mathrm{dez} .2008$. 
RIZZON, L.A. et al. Teores de cátions dos vinhos da Serra Gaúcha. Ciência e Tecnologia de Alimentos, v.28, n.03, p.635-641, 2008. Disponível em: <http:// http://www.scielo.br/ pdf/cta/v28n3/a20v28n3.pdf. Acesso em: 13 maio 2009. doi: 10.1590/S0101-20612008000300020.

SPERKOVÁ, J; SUCHÁNEK, M. Multivariate classification of wines from different Bohemian regions (Czech Republic). Food Chemistry, v.93, p.659-663, 2005. Disponível em: <http:// linkinghub.elsevier.com/retrieve/pii/S0308814604007988>. Acesso em: 13 maio 2009. doi:10.1016/j.foodchem.2004.10.044.

STEFENON, C.A. Atividade antioxidante, composição fenólica e mineral de vinhos espumantes brasileiros.
2006. 85f. Dissertação (Mestrado em Biotecnologia) - Curso de Pós-Graduação em Biotecnologia, Universidade de Caxias do Sul, RS.

STRECK, E. et al. Solos do Rio Grande do Sul. Porto Alegre: EMATER/RS-ASCAR, 2008. 222p.

TONIETTO, J.; CARBONNEAU, A. A multicriteria climatic classification system for grape-growing regions worldwide. Agricultural and Forest Meteorology, v.124, p.81-97, 2004. Disponível em: <http://linkinghub.elsevier.com/retrieve/ pii/S0168192304000115>. Acesso em: 13 maio 2009. doi: 10.1016/j.agrformet.2003.06.001. 